\section{On CALFAN syndrome: report of a patient with a novel variant in SCYL1 gene and recurrent respiratory failure}

To the Editor: We read with great interest the research article published by Lenz and colleagues on CALFAN (low $\gamma$ glutamyl-transferase cholestasis, acute liver failure, and neurodegeneration) syndrome, highlighting the existence of a phenotype with prominent, infantile-onset hepatic involvement and variable neurological phenotype of later onset. ${ }^{1}$

We recently documented an undescribed, but likely pathogenic variant in the SCYL1 (SCY1 like pseudokinase 1) (NM_020680.3) gene in a 7-year-old girl, born at term to nonconsanguineous healthy parents with negative family history, affected by a cerebellar syndrome (tremor, ataxia, and dysmetria), cerebellar atrophy, ocular motility disorder, and intellectual disability, with prominent language involvement.

She had normal early developmental milestones. Since infancy, she has been suffering from frequent respiratory infections, including recurrent episodes of acute respiratory insufficiency with pleural effusion. At 18 months of age, episodes of abdominal distension with normal abdomen ultrasound scans also occurred.

At 23 months of age she started to present with episodic tremor, initially induced by fever. The first consultation at our center (at 2 years and 9 months of age) showed mild axial hypotonia with no weakness. She walked with a broad gait and frequent postural adjustments, and showed dysmetria and diffuse tremor (more prominent in the head and at the distal upper limbs). At 5 years and 6 months of age an ocular motility disorder emerged, with upward gaze palsy and oculomotor apraxia, a worsening of weakness with fatigability was reported by parents, and a distal choreic movement disorder became apparent at the upper limbs. She has been experiencing recurrent episodes of regression (worsening of tremor and postural instability, language regression) during febrile illnesses. However, transaminase analyses during acute febrile illnesses were within normal range. Electroencephalogram (EEG) and electroneurography (ENG) were normal.

Brain magnetic resonance imaging (MRI) documents mild cerebellar atrophy with predominant involvement of the superior vermis and of the lateral portions of the cerebellar hemispheres, stable at follow-up. At the age of 7 years, she has moderate homogeneous hepatomegaly and splenomegaly;
ENG shows severe predominantly axonal motor polyneuropathy.

During follow-up, she underwent the following noncontributory investigations: plasma amino acids, urinary organic acids, acylcarnitines, a-fetoprotein, copper and ceruloplasmin, immunoglobulins, karyotype, array comparative genomic hybridization (array-CGH), molecular analysis of ATM, SPMD1, DYT11, TITF1, ADCY5, APTX, SETX, NPC1 (Niemann-Pick Suspicion Index: 88), and NPC2 genes. She also underwent a negative targeted next-generation sequencing (NGS) panel for ataxia.

Exome sequencing detected the homozygous 1-bp duplication c.1534dupT, p.(Cys512Leufs $\left.{ }^{\star} 8\right)($ NM_020680.3) on the SCYL1 gene, classified as likely pathogenic, which is not reported in the literature, in the Genome Aggregation Database (gnomAD), or in public mutation databases (Human Gene Mutation Database [HGMD], ClinVar). Her parents are heterozygous carriers for this variant.

It results in a 1-bp frameshift leading to a through protein truncation or nonsense-mediated messenger RNA (mRNA) decay. No individuals with homozygous truncating variants in SCYL1 gene have been so far reported in gnomAD, further supporting a disease-causing role.

The SCYL1 gene (MIM $\left.{ }^{\star} 607982\right)$ encodes for a protein likely involved in intracellular transport processes via the Golgi-endoplasmic reticulum pathway and also playing a role in nuclear cytoplasmic transfer RNA (tRNA) transport. ${ }^{2}$ Pathogenic variants in SCYL1 have been associated with autosomal recessive spinocerebellar ataxia-21 (MIM 616719), characterized by early infantile-onset cerebellar ataxia and cerebellar atrophy and recurrent episodes of liver failure in the first decade, frequently triggered by fever, resulting in the development of chronic liver fibrosis with hepato-splenomegaly, later-onset peripheral neuropathy, and possibly mild learning disability. ${ }^{2}$

In summary, we believe that the neurologic phenotype of our patient is consistent with previous descriptions. Interestingly, to the best of our knowledge, no cases with recurrent episodes of respiratory insufficiency with clinical regression have ever been described. Nevertheless, SCYL1 expression has been demonstrated to be ubiquitous, including the lung, suggesting that its function is required by all cells. ${ }^{3}$ Therefore, in line with the frequent involvement of other organs in hereditary cerebellar ataxias, we propose that not only recurrent episodes of hepatic failure, but also recurrent episodes of respiratory failure should be regarded as possible part of the clinical phenotype in patients harboring diseasecausing variants in SCYL1. Further studies are warranted to corroborate our preliminary finding.

In summary, we described a patient harboring a pathogenic variant in SCYL1 gene, presenting with a predominantly 
neurologic phenotype associated with a previously unreported respiratory involvement, thus expanding previous knowledge.

\section{DISCLOSURE}

The authors declare no conflicts of interest.

Carlotta Spagnoli, , $M D^{1}$, Daniele Frattini, , $M D^{1}$, Grazia Gabriella Salerno, , $M D^{1}$ and Carlo Fusco, , $M D^{1,2}$

${ }^{1}$ Department of Pediatrics, Child Neurology Unit, Azienda Unità Sanitaria Locale-IRCCS di Reggio Emilia, Reggio Emilia, Italy; ${ }^{2}$ Pediatric Neurophysiology Laboratory, Azienda Unità Sanitaria Locale-IRCCS di Reggio Emilia, Reggio Emilia, Italy. Correspondence: Daniele Frattini (daniele.frattini@ausl.re.it)

\section{REFERENCES}

1. Lenz D, McClean P, Kansu A. et al. SCYL1 variants cause a syndrome with low $\gamma$-glutamyl-transferase cholestasis, acute liver failure, and neurodegeneration (CALFAN). Genet Med. 2018;20:1255-1265.

2. Schmidt WM, Rutledge SL, Schüle R, et al. Disruptive SCYL1 mutations underlie a syndrome characterized by recurrent episodes of liver failure, peripheral neuropathy, cerebellar atrophy, and ataxia. Am J Hum Genet. 2015;97:855-861.

3. Liu SC, Lane WS, Lienhard GE. Cloning and preliminary characterization of a $105 \mathrm{kDa}$ protein with an N-terminal kinase-like domain. Biochim Biophys Acta. 2000;1517:148-152.

Advance online publication 10 December 2018. doi:10.1038/s41436-018-0389-6 